\title{
The Jet of an Interpolant on a Finite Set
}

\section{Charles Fefferman and Arie Israel}

\begin{abstract}
We study functions $F \in C^{m}\left(\mathbb{R}^{n}\right)$ having norm less than a given constant $M$, and agreeing with a given function $f$ on a finite set $E$.

Let $\Gamma_{f}(S, M)$ denote the convex set formed by taking the $(m-1)$ jets of all such $F$ at a given finite set $S \subset \mathbb{R}^{n}$. We provide an efficient algorithm to compute a convex polyhedron $\tilde{\Gamma}_{\mathrm{f}}(\mathrm{S}, \mathrm{M})$, such that
\end{abstract}

$$
\Gamma_{\mathrm{f}}(\mathrm{S}, \mathrm{cM}) \subset \tilde{\Gamma}_{\mathrm{f}}(\mathrm{S}, \mathrm{M}) \subset \Gamma_{\mathrm{f}}(\mathrm{S}, \mathrm{CM}),
$$

where $\mathrm{c}$ and $\mathrm{C}$ depend only on $\mathrm{m}$ and $\mathrm{n}$.

Fix $m, n \geq 1$, and let $f: E \longrightarrow \mathbb{R}$ be given, where $E \subset \mathbb{R}^{n}$. For a given real number $M>0$, we are interested in functions $F \in C^{m}\left(\mathbb{R}^{n}\right)$ with norm at most $M$, such that $F=f$ on $E$. To understand how $F$ behaves, we fix a finite set $S \subset \mathbb{R}^{n}$, and compute the $(m-1)^{\text {rst }}$ order Taylor polynomial ${ }^{1}$ of $F$ at each point of $S$. What can we say about the resulting family of polynomials?

To answer this question, we introduce some notation and definitions. As usual, $\mathrm{C}^{\mathrm{m}}\left(\mathbb{R}^{\mathrm{n}}\right)$ consists of all $\mathrm{m}$ times continuously differentiable functions $\mathrm{F}: \mathbb{R}^{\mathrm{n}} \longrightarrow \mathbb{R}$ for which the norm

$$
\|F\|=\sup _{x \in \mathbb{R}^{n}} \max _{|\alpha| \leq m}\left|\partial^{\alpha} F(x)\right|
$$

is finite. For $F \in C^{m}\left(\mathbb{R}^{n}\right)$ and $x \in \mathbb{R}^{n}$, we write $J_{x}(F)$ (the "jet" of $F$ at $x$ ) to denote the $(m-1)^{r \text { st }}$ order Taylor polynomial of $F$ at $x$. Thus, $J_{x}(F)$ belongs to $\mathcal{P}$, the vector space of (real-valued) $(m-1)^{\text {rst }}$ degree polynomials on $\mathbb{R}^{n}$.

2000 Mathematics Subject Classification: 49K24, 52A35.

Keywords: Interpolation, jet, algorithm, Whitney extension theorem.

${ }^{1}$ We use $(m-1)$ rst degree Taylor polynomials, since nothing useful can be inferred regarding the $m^{\text {th }}$ derivatives of $F$. 
A "Whitney field" on a finite set $S \subset \mathbb{R}^{n}$ is a family $\vec{P}=\left(\mathrm{P}^{\mathrm{x}}\right)_{\mathrm{x} \in \mathrm{S}}$ of polynomials $\mathrm{P}^{\mathrm{x}} \in \mathcal{P}$, indexed by the points of $S$. We write $\mathrm{Wh}(\mathrm{S})$ to denote the vector space of all Whitney fields on $S$. For $F \in C^{m}\left(\mathbb{R}^{n}\right)$ and $S \subset \mathbb{R}^{n}$ finite, we write $\mathrm{J}_{S}(\mathrm{~F})$ to denote the Whitney field

$$
\mathrm{J}_{S}(\mathrm{~F})=\left(\mathrm{J}_{\mathrm{x}}(\mathrm{F})\right)_{\mathrm{x} \in \mathrm{S}} \in \mathrm{Wh}(\mathrm{S})
$$

Now let $f: E \longrightarrow \mathbb{R}$ with $E \subset \mathbb{R}^{n}$, and let $M>0$ be a real number. For $x \in \mathbb{R}^{n}$, we define

$$
\Gamma_{f}(x, M)=\left\{J_{x}(F): F \in C^{m}\left(\mathbb{R}^{n}\right),\|F\| \leq M, F=f \text { on } E\right\} \subset \mathcal{P} .
$$

More generally, for finite $S \subset \mathbb{R}^{n}$, we define

$$
\Gamma_{\mathrm{f}}(\mathrm{S}, \mathrm{M})=\left\{\mathrm{J}_{\mathrm{S}}(\mathrm{F}): \mathrm{F} \in \mathrm{C}^{\mathrm{m}}\left(\mathbb{R}^{\mathrm{n}}\right),\|\mathrm{F}\| \leq \mathrm{M}, \mathrm{F}=\mathrm{f} \text { on } \mathrm{E}\right\} \subset \mathrm{Wh}(\mathrm{S}) .
$$

Thus, $\Gamma_{f}(x, M)$ and $\Gamma_{f}(S, M)$ are (possibly empty) convex sets.

Fefferman-Klartag [2] gave an efficient algorithm to compute a convex polyhedron $\tilde{\Gamma}_{\mathrm{f}}(\mathrm{x}, \mathrm{M}) \subset \mathcal{P}$ that approximates $\Gamma_{\mathrm{f}}(\chi, M)$.

Our purpose here is to compute a convex polyhedron $\widetilde{\Gamma}_{f}(S, M) \subset \mathrm{Wh}(S)$ that approximates $\Gamma_{\mathrm{f}}(S, M)$.

Our algorithms, here and in $[2,3]$, are to be run on an (idealized) digital computer with standard von Neumann architecture [6], able to store and perform arithmetic operations on exact real numbers. See [2] for a more careful discussion of our model of computation. We are interested in the number of elementary operations used by our algorithms, and also in the number of memory cells required. (We suppose each memory cell can hold one real number.) We refer to the number of elementary operations, and the number of memory cells, as the "work" and "storage", respectively.

We will be working below with finite sets $E, S \subset \mathbb{R}^{n}$. We write $N_{E}$ and $N_{S}$ to denote the number of elements of the sets $E$ and $S$, respectively.

We write $\mathbf{c}, C, C^{\prime}$, etc., to denote constants depending only on $m$ and $n$. These symbols may denote different constants in different occurrences.

One of the main results of Fefferman-Klartag [2] is as follows.

Theorem 1. Fix $\mathrm{m}, \mathrm{n} \geq 1$. Given $\mathrm{f}: \mathrm{E} \rightarrow \mathbb{R}$ with $\mathrm{E} \subset \mathbb{R}^{\mathrm{n}}$ finite, and given $\mathrm{M}>0$ real, we can perform one-time work less than $\mathrm{CN}_{\mathrm{E}} \log \left(\mathrm{N}_{\mathrm{E}}+1\right)$ using storage $\mathrm{CN}_{\mathrm{E}}$, after which we can answer queries as follows.

A query consists of a point $\mathrm{x} \in \mathbb{R}^{\mathrm{n}}$. In response to a query $\mathrm{x}$, we produce a (possibly empty) convex polyhedron $\widetilde{\Gamma}_{\mathrm{f}}(\mathrm{x}, \mathrm{M}) \subset \mathcal{P}$, defined by at most $\mathrm{C}$ linear constraints, such that

$$
\Gamma_{\mathrm{f}}(\mathrm{x}, \mathrm{cM}) \subset \tilde{\Gamma}_{\mathrm{f}}(\mathrm{x}, \mathrm{M}) \subset \Gamma_{\mathrm{f}}(\mathrm{x}, \mathrm{CM}) .
$$

The work used to answer a query is at most $\mathrm{C} \log \left(\mathrm{N}_{\mathrm{E}}+1\right)$. 
The main result of this paper is the following generalization of Theorem 1 .

Theorem 2. Fix $\mathrm{m}, \mathrm{n} \geq 1$. Given $\mathrm{f}: \mathrm{E} \rightarrow \mathbb{R}$ with $\mathrm{E} \subset \mathbb{R}^{\mathrm{n}}$ finite, and given $M>0$ real, we can perform one-time work less than $C \mathrm{~N}_{\mathrm{E}} \log \left(\mathrm{N}_{\mathrm{E}}+1\right)$ using storage $\mathrm{CN}_{\mathrm{E}}$, after which we can answer queries as follows.

$A$ query consists of a finite set $\mathrm{S} \subset \mathbb{R}^{\mathrm{n}}$. In response to a query $\mathrm{S}$, we produce a (possibly empty) convex polyhedron $\tilde{\Gamma}_{\mathrm{f}}(\mathrm{S}, \mathrm{M}) \subset \mathrm{Wh}(\mathrm{S})$, defined by at most $\mathrm{CN}_{\mathrm{S}}$ linear constraints, such that

$$
\Gamma_{\mathrm{f}}(\mathrm{S}, \mathrm{cM}) \subset \tilde{\Gamma}_{\mathrm{f}}(\mathrm{S}, \mathrm{M}) \subset \Gamma_{\mathrm{f}}(\mathrm{S}, \mathrm{CM}) .
$$

The work used to answer a query is at most $\mathrm{CN}_{\mathrm{S}} \log \left(\mathrm{N}_{\mathrm{E}}+\mathrm{N}_{\mathrm{S}}\right)$.

We were pleasantly surprised to learn that Theorem 2 follows easily from Theorem 1, thanks to a lemma in [3] and the following simple observation.

Lemma 1. Let $\mathrm{f}: \mathrm{E} \longrightarrow \mathbb{R}$ with $\mathrm{E} \subset \mathbb{R}^{n}$, let $\mathrm{M}>0$ be a real number, and let $\overrightarrow{\mathrm{P}}=\left(\mathrm{P}^{\mathrm{x}}\right)_{\mathrm{x} \in \mathrm{S}} \in \mathrm{Wh}(\mathrm{S})$, with $\mathrm{S} \subset \mathbb{R}^{\mathrm{n}}$ finite. Suppose that, for each $\mathrm{x} \in \mathrm{S}$, there exists $\mathrm{F}^{\mathrm{x}} \in \mathrm{C}^{\mathrm{m}}\left(\mathbb{R}^{\mathrm{n}}\right)$, such that

$$
\left\|F^{x}\right\| \leq M, F^{x}=f \text { on } E \text {, and } J_{x}\left(F^{x}\right)=P^{x} .
$$

Suppose also that

(2) $\left|\partial^{\alpha}\left(\mathrm{P}^{\mathrm{x}}-\mathrm{P}^{\mathrm{y}}\right)(\mathrm{y})\right| \leq \mathrm{M}|\mathrm{x}-\mathrm{y}|^{\mathrm{m}-|\alpha|}$ for $|\alpha| \leq \mathrm{m}-1, \mathrm{x}, \mathrm{y} \in \mathrm{S}$ distinct.

Then there exists $\mathrm{F} \in \mathrm{C}^{\mathrm{m}}\left(\mathbb{R}^{\mathrm{n}}\right)$ such that

$$
\|\mathrm{F}\| \leq \mathrm{CM}, \mathrm{F}=\mathrm{f} \text { on } \mathrm{E} \text {, and } \mathrm{J}_{\mathrm{S}}(\mathrm{F})=\overrightarrow{\mathrm{P}} \text {. }
$$

The proof of Lemma 1 will be given below. The lemma from [3] used here is as follows.

Lemma 2. Given a finite set $\mathrm{S} \subset \mathbb{R}^{n}$, we can compute a finite list $\left(x_{1}^{\prime}, x_{1}^{\prime \prime}\right) \ldots$ $\left(x_{\mathrm{L}}^{\prime}, x_{\mathrm{L}}^{\prime \prime}\right) \in \mathrm{S} \times \mathrm{S} \backslash$ Diagonal, with

$$
\mathrm{L} \leq \mathrm{CN}_{\mathrm{S}}
$$

such that the following holds. Let $\overrightarrow{\mathrm{P}}=\left(\mathrm{P}^{\mathrm{x}}\right)_{\mathrm{x} \in \mathrm{S}} \in \mathrm{Wh}(\mathrm{S})$, and let $\mathrm{M}>0$ be a real number. If

$$
\left|\partial^{\alpha}\left(\mathrm{P}^{x_{\ell}^{\prime}}-\mathrm{P}^{x_{\ell}^{\prime \prime}}\right)\left(x_{\ell}^{\prime \prime}\right)\right| \leq M\left|x_{\ell}^{\prime}-x_{\ell}^{\prime \prime}\right|^{m-|\alpha|} \text { for }|\alpha| \leq m-1,1 \leq \ell \leq \mathrm{L},
$$

then

$$
\left|\partial^{\alpha}\left(\mathrm{P}^{\mathrm{x}}-\mathrm{P}^{\mathrm{y}}\right)(\mathrm{y})\right| \leq \mathrm{CM}|\mathrm{x}-\mathrm{y}|^{\mathrm{m}-|\alpha|} \text { for }|\alpha| \leq \mathrm{m}-1, \mathrm{x}, \mathrm{y} \in \mathrm{S} \text { distinct. }
$$

The computation of the pairs $\left(x_{1}^{\prime}, x_{1}^{\prime \prime}\right), \cdots,\left(x_{\mathrm{L}}^{\prime}, \chi_{\mathrm{L}}^{\prime \prime}\right)$ requires work at most $\mathrm{CN}_{\mathrm{S}} \log \left(\mathrm{N}_{\mathrm{S}}+2\right)$, and storage at most $\mathrm{CN}_{\mathrm{S}}$.

Here, the $\left(x_{\ell}^{\prime}, x_{\ell}^{\prime \prime}\right)$ are "representatives" arising from a "well-separated pairs decomposition" of $S \times S \backslash$ Diagonal. See Callahan-Kosaraju [1], and compare with the computation of Lipschitz norms in Har-Peled-Mendel [4]. 
In a moment, we prove our new results, namely Lemma 1 and Theorem 2. First, it is a pleasure to thank Bo'az Klartag, Garving (Kevin) Luli and Nahum Zobin for valuable conversations, and Gerree Pecht for $\mathrm{HT}_{\mathrm{E}} \mathrm{Xing}$ our manuscript to her usual impeccable standards.

Let us see how to prove Theorem 2, once we know Theorem 1 and Lemmas 1 and 2. Given $M>0$ and $f: E \rightarrow \mathbb{R}$, we perform the same one-time work as in Theorem 1. Now suppose we are given a query $S \subset \mathbb{R}^{n}$. We compute the list $\left(x_{1}^{\prime}, x_{1}^{\prime \prime}\right), \ldots,\left(x_{\mathrm{L}}^{\prime}, x_{\mathrm{L}}^{\prime \prime}\right)$ as in Lemma 2. Also, for each $x \in S$ we compute $\tilde{\Gamma}_{f}(x, M)$, as in Theorem 1 . We define $\tilde{\Gamma}_{f}(S, M)$ to consist of all Whitney fields $\vec{P}=\left(\mathrm{P}^{\mathrm{x}}\right)_{\mathrm{x} \in \mathrm{S}}$, such that: $\mathrm{P}^{\mathrm{x}} \in \tilde{\Gamma}_{\mathrm{f}}(\mathrm{x}, \mathrm{M})$ for each $x \in S$; and

$$
\left|\partial^{\alpha}\left(\mathrm{P}^{\mathrm{x}_{\ell}^{\prime}}-\mathrm{P}^{\mathrm{x}_{\ell}^{\prime \prime}}\right)\left(\mathrm{x}_{\ell}^{\prime \prime}\right)\right| \leq M\left|\mathrm{x}_{\ell}^{\prime}-\mathrm{x}_{\ell}^{\prime \prime}\right|^{\mathrm{m}-|\alpha|} \text { for }|\alpha| \leq \mathrm{m}, 1 \leq \ell \leq \mathrm{L} .
$$

Recall that each $\tilde{\Gamma}_{\mathrm{f}}(x, M)$ is a convex polyhedron, defined by at most $\mathrm{C}$ linear constraints. Hence, by $(4), \tilde{\Gamma}_{\mathrm{f}}(\mathrm{S}, \mathbf{M})$ is a convex polyhedron defined by at most $\mathrm{CN}_{\mathrm{S}}$ constraints. Moreover, one checks easily that the work and storage used to compute $\tilde{\Gamma}_{\mathrm{f}}(\mathrm{S}, \mathrm{M})$ are as asserted in Theorem 2. Finally, the desired inclusions

$$
\Gamma_{\mathrm{f}}(\mathrm{S}, \mathrm{cM}) \subset \tilde{\Gamma}_{\mathrm{f}}(\mathrm{S}, \mathrm{M}) \subset \Gamma_{\mathrm{f}}(\mathrm{S}, \mathrm{CM})
$$

follow trivially from Theorem 1, Lemmas 1 and 2, Taylor's theorem, and the definitions of $\Gamma_{f}(x, M), \Gamma_{f}(S, M)$ and $\tilde{\Gamma}_{f}(S, M)$. Thus, Theorem 2 follows from Theorem 1 and Lemmas 1 and 2.

Since Theorem 1 and Lemma 2 are known, our task here reduces to the proof of Lemma 1, which we now provide.

Thus, let $f: E \rightarrow \mathbb{R}, M>0, \vec{P}=\left(P^{x}\right)_{x \in S}$ and $F^{x}$ (each $x \in S$ ) satisfy the hypotheses of Lemma 1 . We must construct an $F \in C^{m}\left(\mathbb{R}^{n}\right)$ satisfying (3).

To do so, we adapt the standard proof $[5,7]$ of the Whitney extension theorem, which we now recall.

First, we define a collection $\Omega$ of "Whitney cubes" Q. The Whitney cubes form a partition of $\mathbb{R}^{n} \backslash S$. Each $Q \in \Omega$ satisfies

$$
\mathrm{c} \delta_{\mathrm{Q}} \leq \operatorname{distance}\left(\mathrm{S}, \mathrm{Q}^{*}\right) \leq \mathrm{C} \delta_{\mathrm{Q}} \text {. }
$$

Here, $\delta_{\mathrm{Q}}$ denotes the side-length of $\mathrm{Q}$, and $\mathrm{Q}^{*}$ denotes the cube $\mathrm{Q}$ dilated about its center by a factor of 3 .

The Whitney cubes have "good geometry": If $\mathrm{Q}^{*} \cap \widehat{\mathrm{Q}}^{*} \neq \emptyset$ with $\mathrm{Q}, \hat{\mathrm{Q}} \in \Omega$, then $\mathrm{c} \delta_{\mathrm{Q}} \leq \delta_{\hat{\mathrm{Q}}} \leq \mathrm{C} \delta_{\mathrm{Q}}$.

Next, for each $\mathrm{Q} \in \Omega$, we pick a "representative" $x_{Q} \in S$, such that distance $\left(\mathrm{x}_{\mathrm{Q}}, \mathrm{Q}^{*}\right) \leq \mathrm{C} \delta_{\mathrm{Q}}$.

We then introduce the "Whitney partition of unity" $1=\sum_{\mathrm{Q} \in \Omega} \theta_{\mathrm{Q}}$ on $\mathbb{R}^{n} \backslash \mathrm{S}$. Here, each $\theta_{\mathrm{Q}}$ belongs to $\mathrm{C}^{\mathrm{m}}\left(\mathbb{R}^{\mathrm{n}}\right)$ and satisfies supp $\theta_{\mathrm{Q}} \subset \mathrm{Q}^{*}$; and $\left|\partial^{\alpha} \theta_{\mathrm{Q}}\right| \leq \mathrm{C} \delta_{\mathrm{Q}}^{-|\alpha|}$ on $\mathbb{R}^{n}$, for $|\alpha| \leq \mathrm{m}$. 
Whitney's proof then defines

$$
\tilde{\mathrm{F}}=\sum_{\mathrm{Q} \in \Omega} \theta_{\mathrm{Q}} \cdot \mathrm{P}^{\mathrm{x}_{\mathrm{Q}}} \text { on } \mathbb{R}^{\mathrm{n}} \backslash \mathrm{S}, \tilde{\mathrm{F}}(\mathrm{x})=\mathrm{P}^{\mathrm{x}}(\mathrm{x}) \text { for } \mathrm{x} \in \mathrm{S} .
$$

The derivatives of $\tilde{\mathrm{F}}$ up to order $\mathrm{m}$ can be controlled on $\mathbb{R}^{n} \backslash \mathrm{S}$, thanks to the estimate

$$
\left|\partial^{\alpha}\left(\mathrm{P}^{\mathrm{x}_{\mathrm{Q}}}-\mathrm{P}^{\mathrm{x}_{\hat{\mathrm{Q}}}}\right)\right| \leq \mathrm{CM} \delta_{\mathrm{Q}}^{\mathrm{m}-|\alpha|} \text { on } \mathrm{Q}^{*} \cap \hat{\mathrm{Q}}^{*} \text {, for }|\alpha| \leq \mathrm{m} .
$$

Estimate (6) follows easily from hypothesis (2).

We will slightly modify Whitney's proof by defining

$$
F=\sum_{Q \in \Omega} \theta_{Q} \cdot F^{x_{Q}} \text { on } \mathbb{R}^{n} \backslash S, F(x)=F^{x}(x) \text { for } x \in S,
$$

in place of (5). From hypothesis (1) and Taylor's theorem, we see that

$$
\left|\partial^{\alpha}\left(\mathrm{F}^{\mathrm{x}_{\mathrm{Q}}}-\mathrm{P}^{\mathrm{x}_{\mathrm{Q}}}\right)\right| \leq \mathrm{CM} \delta_{\mathrm{Q}}^{\mathrm{m}-|\alpha|} \text { on } \mathrm{Q}^{*} \text {, for }|\alpha| \leq \mathrm{m} .
$$

Similarly

$$
\left|\partial^{\alpha}\left(\mathrm{F}^{\mathrm{x}_{\mathrm{Q}}}-\mathrm{P}^{\mathrm{x}_{\mathrm{Q}}}\right)\right| \leq \mathrm{CM} \delta_{\hat{\mathrm{Q}}}^{\mathrm{m}-|\alpha|} \text { on } \hat{\mathrm{Q}}^{*} \text {, for }|\alpha| \leq \mathrm{m} .
$$

Since $c \delta_{\mathrm{Q}} \leq \delta_{\widehat{\mathrm{Q}}} \leq \mathrm{C} \delta_{\mathrm{Q}}$ for $\mathrm{Q}^{*} \cap \widehat{\mathrm{Q}}^{*} \neq \emptyset$, we learn from (6), (8), (9) that

$$
\left|\partial^{\alpha}\left(\mathrm{F}^{\mathrm{x}_{\mathrm{Q}}}-\mathrm{F}^{\mathrm{x}_{\hat{Q}}}\right)\right| \leq \mathrm{CM} \delta_{\mathrm{Q}}^{\mathrm{m}-|\alpha|} \text { on } \mathrm{Q}^{*} \cap \hat{\mathrm{Q}}^{*} \text {, for }|\alpha| \leq \mathrm{m} \text {. }
$$

Estimate (10) is useful when $\delta_{\mathrm{Q}} \leq 1$. For $\delta_{\mathrm{Q}}>1$, we use instead the estimate

$$
\left|\partial^{\alpha}\left(F^{x_{Q}}-F^{x_{Q}}\right)\right| \leq C M \text { on } Q^{*} \cap \hat{Q} \text { for }|\alpha| \leq m ;
$$

this estimate is immediate from hypothesis (1) .

By using (10) and (11) in place of (6), we learn from Whitney's classic argument that

$$
\mathrm{F} \in \mathrm{C}_{\text {loc }}^{\mathrm{m}}\left(\mathbb{R}^{\mathrm{n}} \backslash \mathrm{S}\right) \text {, and }\left|\partial^{\alpha} \mathrm{F}\right| \leq \mathrm{CM} \text { on } \mathbb{R}^{\mathrm{n}} \backslash \mathrm{S} \text {, for }|\alpha| \leq \mathrm{m} .
$$

Also, from (1) and (7), we see at once that

$$
F=f \text { on } E \backslash S \text {. }
$$

On the other hand, let $x \in S$. Since $S$ is finite, $x$ has a small open neighborhood $\mathrm{U}_{x}$ with the following property: Let $\mathrm{Q} \in \Omega$, and suppose $\mathrm{Q}^{*} \cap \mathrm{U}_{\mathrm{x}} \neq \emptyset$. Then $\mathrm{x}_{\mathrm{Q}}=\mathrm{x}$.

Hence, by (7), we have $\mathrm{F}=\mathrm{F}^{\mathrm{x}}$ on $\mathrm{U}_{\mathrm{x}}$. 
Hypothesis (1) therefore tells us that

$$
\begin{gathered}
F \in C_{\ell o c}^{m}\left(U_{x}\right),\left|\partial^{\alpha} F\right| \leq M \text { on } U_{x} \text { for }|\alpha| \leq m, J_{x}(F)=P^{x}, \text { and } \\
F(x)=f(x) \text { if } x \in E \cap S .
\end{gathered}
$$

The desired properties (3) of the function $F$ now follow trivially from (12)-(15). The proof of Lemma 1 is complete

\section{References}

[1] Callahan, P. B. and Kosaraju, S. R.: A decomposition of multidimensional point sets with applications to k-nearest-neighbors and n-body potential fields. J. Assoc. Comput. Mach. 42 (1995), no. 1, 67-90.

[2] Fefferman, C. and Klartag, B.: Fitting a $\mathrm{C}^{\mathrm{m}}$-smooth function to data. Part I, Ann. of Math. (2) 169 (2009), 315-346. Part II, Rev. Mat. Iberoam. 25 (2009), 49-273.

[3] Fefferman, C.: Fitting a $\mathrm{C}^{\mathrm{m}}$-smooth function to data. III. Ann of Math. (2) $\mathbf{1 7 0}$ (2009), no. 1, 427-441.

[4] Har-Peled, S. and Mendel, M.: Fast construction of nets in lowdimensional metrics, and their applications. SIAM J. Comput. 35 (2006), no. 5, 1148-1184 (electronic).

[5] Malgrange, B.: Ideals of differentiable functions. Oxford University Press, London, 1967.

[6] von Neumann, J.: First draft of a report on the EDVAC. Contract No. W670-ORD-492, Moore School of Electrical Engineering, Univ. Pennsylvania, 1945. Reprinted in IEEE Ann. Hist. Comput. 15 (1993), no. 4, 27-75.

[7] Stein, E. M.: Singular integrals and differentiability properties of functions. Princeton Math. Series 30. Princeton Univ. Press, Princeton, 1970.

Recibido: 17 de septiembre de 2009

Charles Fefferman

Department of Mathematics

Princeton University, Fine Hall

Washington Road, Princeton, New Jersey 08544

cf@math.princeton.edu

Arie Israel

Department of Mathematics

Princeton University, Fine Hall

Washington Road, Princeton, New Jersey 08544

aisrael@math.princeton. edu

The authors are supported by grants DMS-0601025 \& ONR-N00014-08-1-0678. 